Supporting Information

for

\title{
Binding Orientation of a Ruthenium-Based Water Oxidation Catalyst on a CdS QD Surface Revealed by NMR Spectroscopy
}

Orion M. Pearce, Jeremiah S. Duncan, Bimala Lama, Gordana Dukovic," Niels H. Damrauer*

Department of Chemistry and Renewable and Sustainable Energy Institute (RASEI), University of Colorado Boulder, Boulder, Colorado 80309, United States

\section{Table of Contents:}

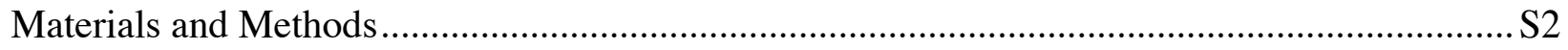

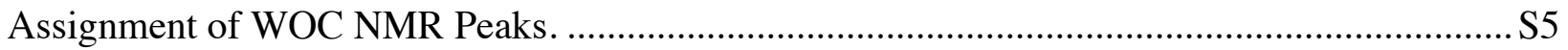

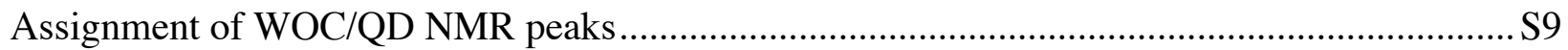

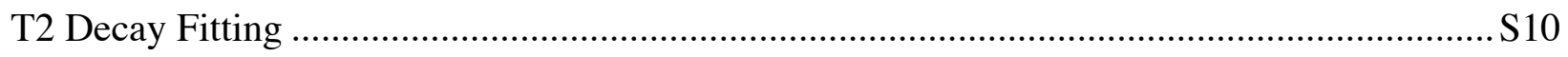

Chemical Shifts and T2 Lifetimes by Proton ............................................................................ S10

Estimation of $\mathrm{T}_{2} *$ through NMR Peak Linewidth Fitting .........................................................S11 


\section{Materials and Methods}

CdS QDs were synthesized following a previously reported method. ${ }^{1}$ All steps were performed under an inert argon atmosphere using standard Schlenk line or glovebox techniques. A solution of $0.10 \mathrm{M}$ sulfur in 1-octadecene (ODE; Sigma Aldrich, 90\%) was prepared (typical amounts: $0.056 \mathrm{~g} \mathrm{~S}_{8}, 14 \mathrm{~g} \mathrm{ODE}$ ). A $0.10 \mathrm{M}$ solution of cadmium oleate was produced by heating under argon $0.256 \mathrm{~g}$ of $\mathrm{CdO}$ (99.99\%, Aldrich), $14 \mathrm{~mL}$ of ODE, and $6.4 \mathrm{~mL}$ of oleic acid (OA; Sigma Aldrich, $90 \%$ ) to $250^{\circ} \mathrm{C}$ in a $50 \mathrm{~mL}$ 3-neck flask fitted with a reflux condenser. After the solution turned clear the solution was allowed to cool to $60{ }^{\circ} \mathrm{C}$. A second 3-neck flask fitted with reflux condenser was charged with $6 \mathrm{~mL}$ of ODE and $4 \mathrm{~mL}$ of cadmium oleate solution and evacuated for 30 minutes at $90^{\circ} \mathrm{C}$. This solution was then heated to $260{ }^{\circ} \mathrm{C}, 2 \mathrm{~mL}$ of $0.10 \mathrm{M}$ sulfur solution was injected, and the reaction mixture was allowed to cool to $220^{\circ} \mathrm{C}$. Three minutes later, $0.5 \mathrm{~mL}$ of $0.10 \mathrm{M}$ cadmium oleate solution was injected. Ten subsequent $0.5 \mathrm{~mL}$ additions of precursors, alternating between sulfur and cadmium oleate solutions, were performed every minute, while reaction temperature was maintained near $220^{\circ} \mathrm{C}$. A minimal amount of reaction solution was removed via syringe after approximately 3 injections and added to $2 \mathrm{~mL}$ of ODE in a cuvette and analyzed by UV-Vis spectroscopy. The final injection was cadmium oleate. Following this addition, the solution was rapidly cooled by immersion in a room temperature oil bath. The solution was transferred by syringe to an Ar-purged scintillation vial and the crude mixture was pumped into an Ar-filled glovebox. CdS QDs were purified using a liquid-liquid extraction with a 3:1 ratio of methanol (HPLC grade, Sigma Aldrich) to the reaction mixture. The white methanol layer was removed, and minimal volume of acetone $(\sim 3 \mathrm{~mL})$ was added to the yellow ODE layer to precipitate the CdS QDs. The 
sample was centrifuged, the supernatant discarded, and the yellow pellet dissolved in a minimal amount of anhydrous toluene $(\sim 1.5 \mathrm{~mL})$. This precipitation / dispersion purification process was repeated 2 times. The size distribution was narrowed using sizeselective precipitation. Isopropanol was added dropwise until a precipitate formed, followed by centrifuging and decanting. This process was repeated 5 times, with a narrow size distribution of QD's accounting for a majority of the product being extracted after the third addition. The size-selected sample was precipitated with methanol, centrifuged, and redispersed in anhydrous toluene. The resulting QDs had the first exciton peak maximum at $462 \mathrm{~nm}$, corresponding to an average diameter of $5.8 \mathrm{~nm}$ and a molar absorptivity of $1.2 \times 10^{6} \mathrm{M}^{-1} \mathrm{~cm}^{-1}$ based on the sizing curves from Yu et al. ${ }^{2}$

QD surface ligands were exchanged to 3-Mercaptopropionate to solubilize the QDs in methanol. This was achieved based on a procedure from the previous report ${ }^{3}$ of interaction between this WOC and CdS QD but with the following modifications. The typical volume of oleate-capped QDs used for preparation of ligand exchanges was increased 5 to 10 -fold, ( $500 \mu \mathrm{L}-1 \mathrm{~mL})$ and the volume of ligand exchange solution was doubled $(2 \mathrm{~mL})$. Following precipitation with toluene and re-suspension in methanol, the particles were precipitated again using a second addition of toluene in minimal volume. The supernatant was decanted, several drops of methanol were added, and the solution was dried in a vacuum chamber overnight. This procedure typically resulted in the removal of most of the toluene, but a residual solvent peak in the proton NMR was still noticeable in some cases. The dried pellet was redispersed in a minimal volume of deuterated methanol and its concentration determined using UV-Visible spectroscopy. 
The WOC was synthesized as described previously ${ }^{3}$ and the same product batch was used here. All samples used for NMR studies were dissolved in deuterated solvents in an Ar filled glovebox shortly prior to use.

For all NMR experiments, $500-700 \mu \mathrm{L}$ solutions were made under Ar. Samples containing QDs were sealed using screw-caps in airtight tubes. Typical concentrations for these experiments were $35 \mu \mathrm{M}$ for QDs and/or $350 \mu \mathrm{M}$ WOC in $\mathrm{CD}_{3} \mathrm{OD}$. Experiments involving WOC without QDs were performed in deuterated $\mathrm{CO}\left(\mathrm{CD}_{3}\right)_{2}$ to minimize transesterification. ${ }^{3}$ For routine NMR spectroscopy, ${ }^{1} \mathrm{H}$ NMR spectra were acquired on a Bruker advance-III spectrometer operating at a ${ }^{1} \mathrm{H}$ frequency of $300 \mathrm{MHz}$ equipped with smart broadband probe at room temperature ( $299 \mathrm{~K})$. Samples were referenced to the protic impurity peak of $\left(\mathrm{CD}_{2} \mathrm{H}\right) \mathrm{OD}$. For typical $1 \mathrm{D}^{1} \mathrm{H}$ measurements, $64 \mathrm{k}$ data points were acquired with a spectral width of $21.6 \mathrm{ppm}, 3 \mathrm{~s}$ acquisition time and a $1 \mathrm{~s}$ relaxation delay with pulse width $12.5 \mu \mathrm{s}$.

For two-dimensional NMR experiments including COSY, HSQC, HMBC, TOCSY, and relaxation ( $T_{2}$ lifetime) measurements, an Agilent INOVA 500 spectrometer was used operating at $499.599 \mathrm{MHz}$ and equipped with a $5 \mathrm{~mm}$ triple resonance $\mathrm{HCN}$ probe at 295 K. For ${ }^{1} \mathrm{H}$ NMR data acquisition, 16 scans were collected, each with the following experimental parameters: acquisition time $3.0 \mathrm{~s}$, spectral width $8 \mathrm{kHz}$, pulse width 6.12 $\mu \mathrm{s}$, and relaxation delay $2.0 \mathrm{~s}$. TOCSY spectra were collected using $256 \mathrm{t}_{1}$ increments and spectral widths of $5000 \mathrm{~Hz}$ in both dimensions with an acquisition time of $0.15 \mathrm{~s}$, a relaxation delay of $2.0 \mathrm{~s}$, and a spinlock (MLEV-17) mixing time of $80 \mathrm{~ms}$. Spin-spin relaxation ( $T_{2}$ lifetime) measurements were conducted using the CPMG (Carl-PurcellMeiboom-Gill) pulse sequence. Data were processed using Mnova software. 


\section{Assignment of WOC NMR Peaks.}

Assignment of the WOC NMR spectrum of the catalyst begins with examination of

the ${ }^{1} \mathrm{H}$ NMR spectrum as shown in Figure 1 in the main text. Similar to observations in related WOCs, dmcbpy protons proximal to the halogen display a pronounced downfield shift ${ }^{4}$ and the symmetry disruption means that aromatic protons on this ligand each have an integral of 1 . In contrast, all of the terpyridine protons integrate to 2 , with the exception of a. This proton was easily identified by its triplet splitting pattern.

As protons couple strongly within individual aromatic rings, 4 correlated aromatic spin systems are expected for this molecule corresponding to the two rings of dmcbpy, the central terpyridine ring, and the terminal terpyridine rings. The COSY spectrum shown in Figure $\mathrm{S} 1$ confirms this, with the correlated spin systems being distinguished by color. As we have assigned the farthest downfield resonance to proton $\mathbf{1}$, the protons 2 and 3 were straightforward to assign based on the COSY and ${ }^{1} \mathrm{H}$ spectra, with proton 2 exhibiting a larger $\mathrm{J}$ value than proton 3 . The other dmcbpy ring was assigned similarly, based on the integrals, COSY, and $\mathrm{J}$ values. The terpyridine resonances on the central ring (protons a, b) were trivial to assign from the COSY spectrum and the ${ }^{1} \mathrm{H}$ splitting pattern. Assignment of the protons on the terminal rings of the terpyridine were more challenging as the pairs $\mathbf{c} / \mathbf{f}$ and $\mathbf{d} / \mathbf{e}$ have similar correlations and splitting patterns. Jcoupling analysis suggests that the peak at $8.6 \mathrm{ppm}(\mathrm{J}=8 \mathrm{~Hz})$ is more likely to be meta to the nitrogen $\left(\mathrm{H}_{\mathrm{c}}\right)$ and the peak at $7.7 \mathrm{ppm}(\mathrm{J}=6 \mathrm{~Hz})$ is likely to be ortho to the nitrogen $\left(\mathrm{H}_{\mathrm{f}}\right){ }^{5}$ but the importance of correct assignment merited additional experiments for confirmation. 


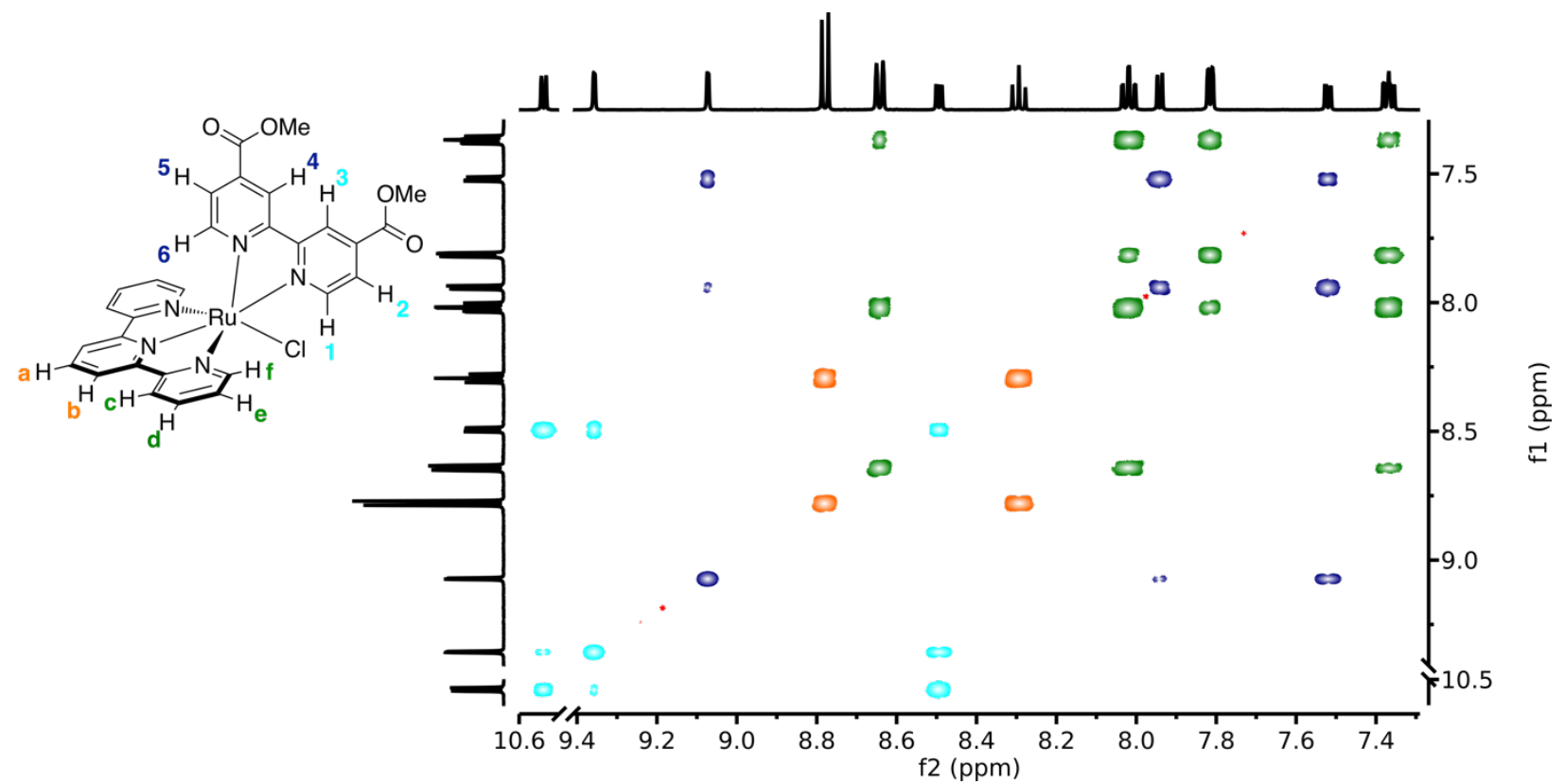

Figure S1. COSY spectrum of the WOC in acetone-d6. Diagonal and cross-peaks are color-coded based upon their correlations, with peaks belonging to the terpyridine highlighted in green and orange and peaks from the bipyridine in blue and teal.

The assignment of these remaining protons was performed using Heteronuclear Single Quantum Coherence (HSQC) and Heteronuclear Multiple Bond Correlation (HMBC) experiments. The HSQC spectrum shown in Figure S2 correlates directly interacting ${ }^{13} \mathrm{C}$ and ${ }^{1} \mathrm{H}$ nuclei, allowing us to assign peaks in the ${ }^{13} \mathrm{C}$ NMR. The HMBC spectrum shown in Figure $\mathrm{S} 3$ correlates ${ }^{13} \mathrm{C}$ and ${ }^{1} \mathrm{H}$ across two or three bonds, with suppression of single bond correlations. Examining the terminal terpyridine ring protons in the HMBC, we see correlations between 3 terpyridine protons and a carbon at 158 ppm. This carbon did not correlate to any protons in the HSQC spectrum, meaning that it is likely the signal of carbons $2\left(\mathbf{C}_{2}\right)$ and $2^{\prime \prime}$ in the terminal terpyridine rings. Proton $\mathbf{e}$ is para to $\mathbf{C}_{2}$ and does not couple to $\mathbf{C}_{2}$ in $\mathrm{HMBC}$, allowing us to assign proton $\mathbf{e}$ to the doublet of doublet of doublets at $7.40 \mathrm{ppm}$. We also assign proton $\mathbf{d}$ to the other doublet 
of doublet of doublets at $8.02 \mathrm{ppm}$. Finally, we assign protons $\mathbf{c}$ and $\mathbf{f}$ based on the COSY, as proton e has a stronger correlation with the doublet of doublets at $7.81 \mathrm{ppm}$ than to the similar signal at $8.64 \mathrm{ppm}$. These assignments follow the same ordering of protons with respect to ${ }^{1} \mathrm{H}$ chemical shift observed in similar terpyridine complexes, as expected by the weakly perturbative interactions between the terpyridine and other ligands. ${ }^{6}$ 


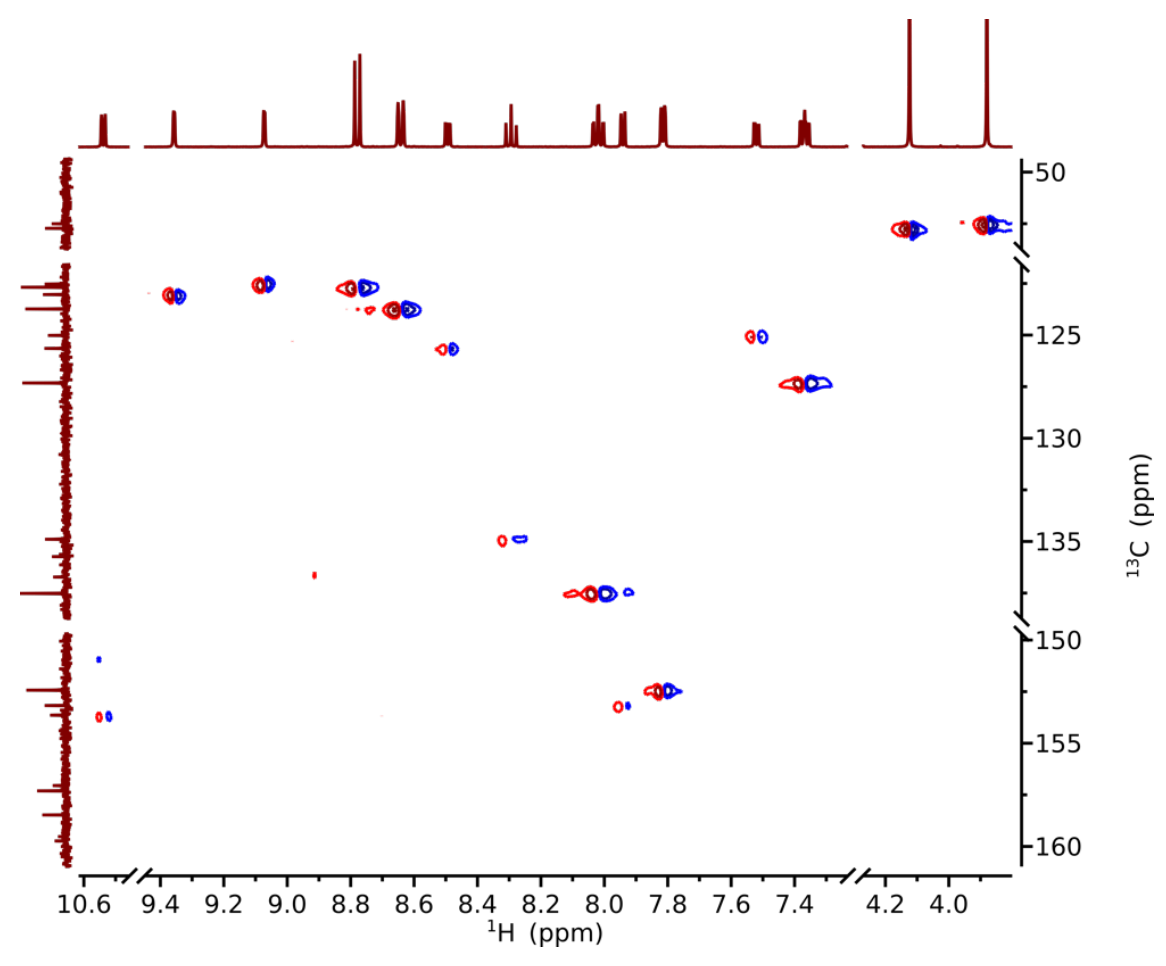

Figure S2. HSQC spectrum of the WOC in acetone-d6.

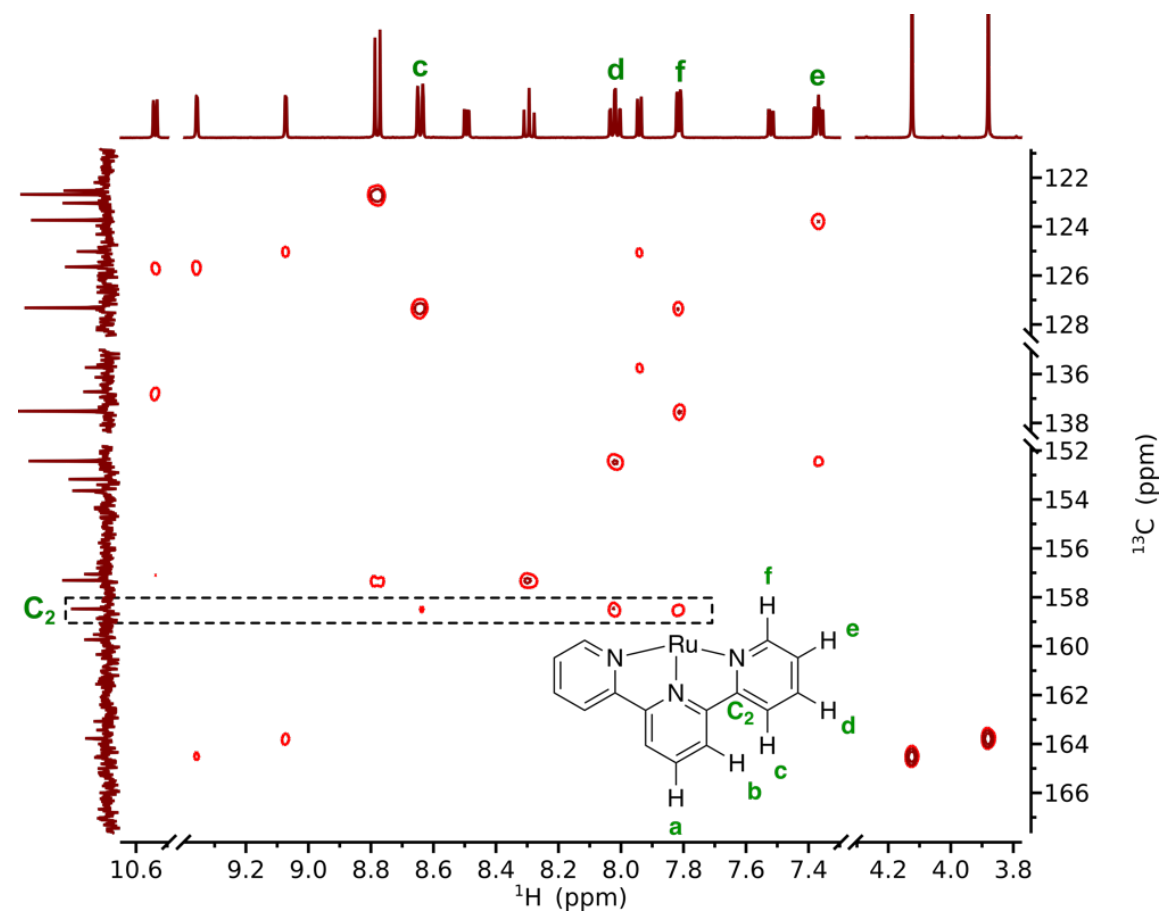

Figure S3. HMBC spectrum of the WOC in acetone-d6. The highlighted peaks are correlations between protons $\boldsymbol{c}, \boldsymbol{d}$, and $\boldsymbol{f}$ with carbons 2 and 2 " in terpyridine. 


\section{Assignment of WOC/QD NMR peaks}

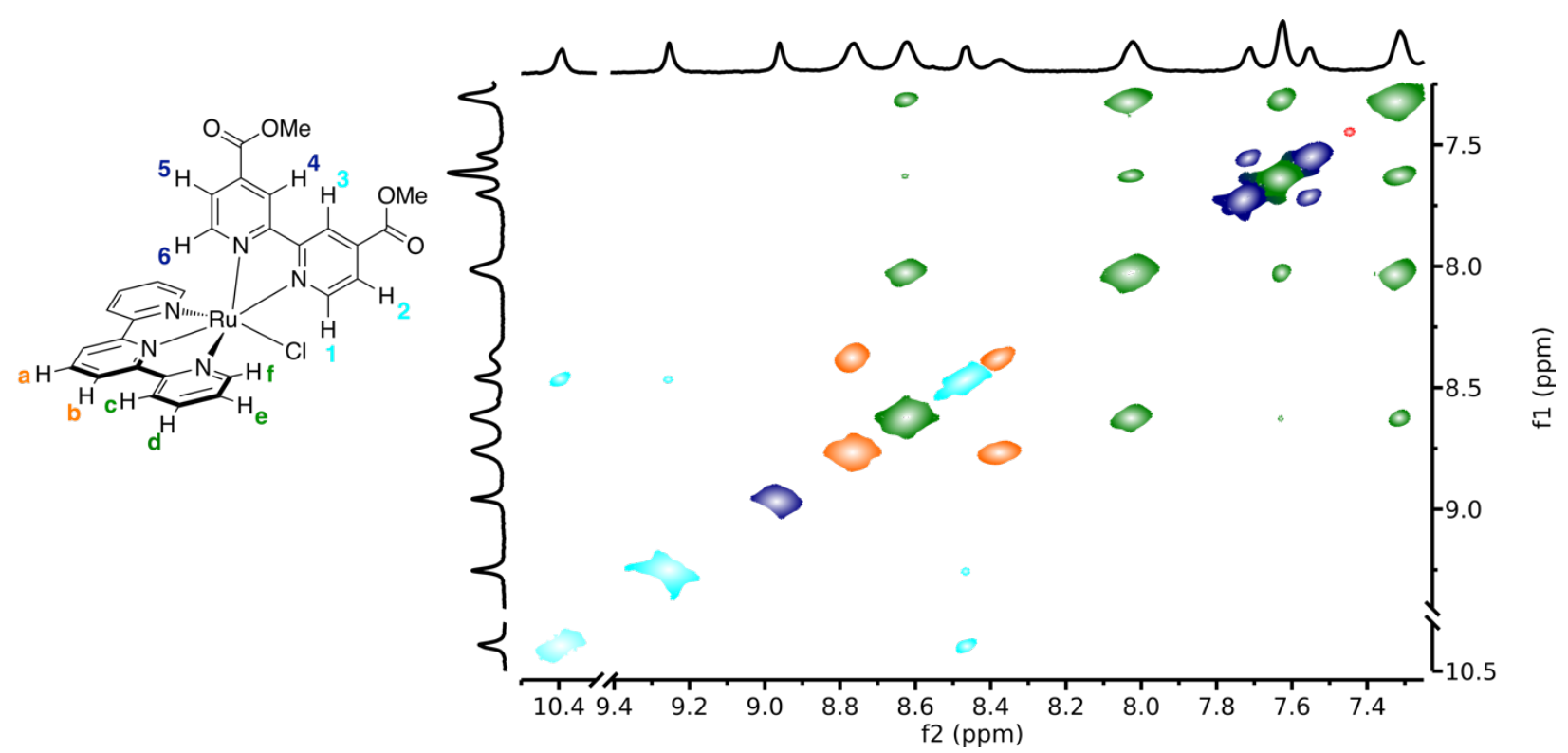

Figure S4. TOCSY spectrum of QDs + WOC in MeOD. Diagonal and cross-peaks are color coded based upon their correlations, with peaks belonging to the terpyridine highlighted in green and orange and peaks from the bipyridine in blue and teal. The similar COSY spectrum of the WOC shown in Figure S1 allows for assignment of each NMR peak of the mixed QD/WOC system to a proton. 
T2 Decay Fitting
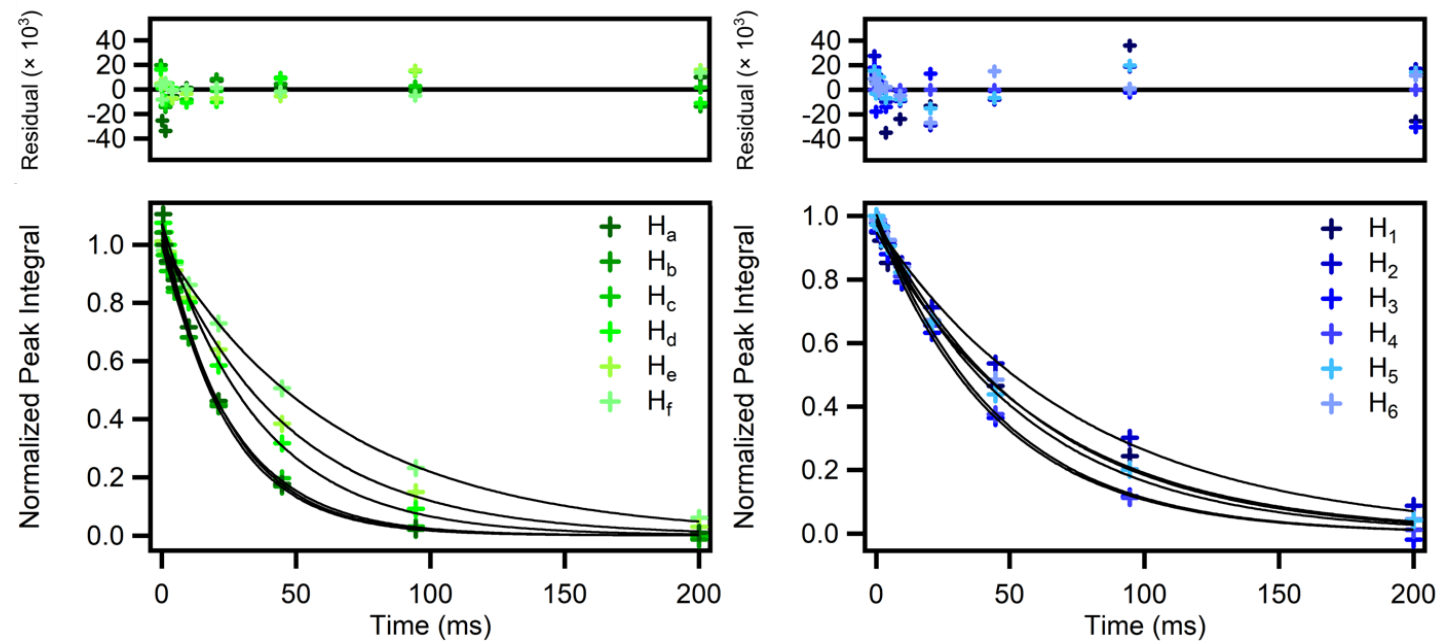

Figure S5. $T_{2}$ decay fitting of the resonances of the $Q D+$ WOC mixture.

\section{Chemical Shifts and T2 Lifetimes by Proton}

Table S1. Summary of chemical shifts (CS) and relaxation parameters for each catalyst peak.

Proton WOC CS QD+WOC $\triangle \mathrm{CS} \quad \mathrm{T}_{2}$ WOC $\mathrm{T}_{2}$ QD+WOC $\mathrm{R}_{2} \mathrm{QD}+\mathrm{WOC}$
(ppm)
CS (ppm) (ppm)
(s)
(ms)
$\left(\mathrm{s}^{-1}\right)$

\begin{tabular}{|l|l|l|l|l|l|l|}
\hline \hline 1 & 10.40 & 10.40 & -0.00 & 2.86 & 62.0 & 16.1 \\
\hline 3 & 9.26 & 9.25 & -0.01 & 1.15 & 44.7 & 22.4 \\
\hline 4 & 8.98 & 8.96 & -0.02 & 1.22 & 45.4 & 22.0 \\
\hline b & 8.69 & 8.76 & 0.08 & 0.92 & 24.2 & 41.3 \\
\hline c & 8.55 & 8.62 & 0.07 & 1.20 & 26.7 & 37.5 \\
\hline 2 & 8.48 & 8.47 & -0.01 & 2.45 & 76.1 & 13.1 \\
\hline a & 8.24 & 8.37 & 0.13 & 1.25 & 24.8 & 40.3 \\
\hline d & 7.96 & 8.92 & 0.07 & 1.92 & 35.9 & 27.9 \\
\hline 6 & 7.70 & 7.72 & 0.02 & 1.99 & 59.2 & 16.9 \\
\hline f & 7.65 & 7.63 & -0.03 & 1.36 & 65.7 & 15.2 \\
\hline 5 & 7.54 & 7.55 & 0.01 & 2.45 & 55.7 & 18.0 \\
\hline e & 7.32 & 7.31 & -0.01 & 1.05 & 46.7 & 21.4 \\
\hline
\end{tabular}




\section{Estimation of $\mathrm{T}_{2}{ }^{*}$ through NMR Peak Linewidth Fitting}

To estimate the combined effects of homogeneous and inhomogeneous broadening in the catalyst spectrum, the peak width was measured for two well-isolated resonances. All aromatic protons on the catalyst are split to some degree by J-coupling to other protons, which greatly complicates the fitting process as the individual peaks of the doublet are no longer resolved after broadening. Furthermore, the peaks are broadened both homogenously and inhomogeneously, leading to a lineshape intermediate between a Lorentzian and Gaussian. ${ }^{7}$

To estimate of $\mathrm{T}_{2}{ }^{*}$ we selected two resonances with small J-coupling (protons 3 , 4). The homogeneous lineshape was approximated based on the free catalyst $\mathrm{J}$-coupling values and the following equation for a lorentzian doublet:

$$
f(x)=\frac{1}{\left(x-x_{1}\right)^{2}+\left(0.5 b_{1}\right)^{2}}+\frac{1}{\left(x-x_{1}-J\right)^{2}+\left(0.5 b_{1}\right)^{2}}
$$

where $x_{1}$ is the chemical shift in $\mathrm{Hz}$ and $\mathrm{b}_{1}$ is the broadness of each individual peak measured as a full-width at half-maximum (FWHM) in $\mathrm{Hz}$. The values of $\mathrm{J}$ and $\mathrm{x}_{1}$ were determined from the catalyst NMR spectrum while the value of $b_{1}$ was determined from the $T_{2}$ value using the equation

$$
b_{1}=\frac{1}{\pi T_{2}}
$$

From this homogeneous lineshape, a homogeneous FWHM was determined and compared to the measured value as shown in Table S2.

Table S2. Comparison between calculated and measured linewidths for selected protons in QDNOC mixture.

\begin{tabular}{l|l|l|}
\multicolumn{1}{l}{ Proton } & \multicolumn{1}{l}{4} & 4 \\
\hline \hline J Coupling (Hz) & 1.8 & 1.8 \\
\hline Calculated FWHM (Hz) & 7.3 & 7.3 \\
\hline Measured FWHM (Hz) & 10.8 & 10.9 \\
\hline Homogeneous Contribution & $68 \%$ & $67 \%$ \\
\hline
\end{tabular}




\section{References}

(1) Peterson, M. D.; Jensen, S. C.; Weinberg, D. J.; Weiss, E. A., Mechanisms for Adsorption of Methyl Viologen on CdS Quantum Dots. ACS Nano 2014, 8, 28262837.

(2) Yu, W. W.; Qu, L.; Guo, W.; Peng, X., Experimental Determination of the Extinction Coefficient of CdTe, CdSe, and CdS Nanocrystals. Chem. Mater. 2003, 15, 2854-2860.

(3) Pearce, O. M.; Duncan, J. S.; Damrauer, N. H.; Dukovic, G., Ultrafast Hole Transfer from CdS Quantum Dots to a Water Oxidation Catalyst. J. Phys. Chem. C 2018, 122, 17559-17565.

(4) Kaveevivitchai, N.; Zong, R.; Tseng, H.-W.; Chitta, R.; Thummel, R. P., Further Observations on Water Oxidation Catalyzed by Mononuclear Ru(II) Complexes. Inorg. Chem. 2012, 51, 2930-2939.

(5) Robert, M. S.; Francis, X. W.; David, J. K., Spectrometric Identification of Organic Compounds. 7th ed.; Wiley: 2005; p 106.

(6) Wasylenko, D. J.; Ganesamoorthy, C.; Koivisto, B. D.; Henderson, M. A.; Berlinguette, C. P., Insight into Water Oxidation by Mononuclear Polypyridyl Ru Catalysts. Inorg. Chem. 2010, 49, 2202-2209.

(7) Higinbotham, J.; Marshall, I., NMR lineshapes and lineshape fitting procedures. In Annual Reports on NMR Spectroscopy, Academic Press: 2001; Vol. 43, pp 59120. 